\title{
A Drumming Based Multimodal Mobile Game Interface
}

\author{
Ahmet Ozkul ${ }^{1}$, Hatice Kose ${ }^{2}$, Rabia Yorganci ${ }^{3}$, Gokhan Ince ${ }^{* 4}$
}

\begin{abstract}
Submitted: 07/11/2019 Accepted: 07/02/2020
Abstract: This study introduces preliminary results from an interactive drumming game, which presents a generic mobile interface. It inspects human-computer interface techniques using body language and aims to support children's mental and psychological progress. The main goal of this work is to focus on the design aspects and verify that a multimodal mobile interface based on touch, visual and auditory stimuli can be used to test children's imitation skills, turn-taking and memory capacities efficiently. The game is tested with a group of children living in the countryside, who were naive to technology and another group of children living in a metropolitan capital city, Ankara, who were the same age but actively using computer/mobile games. The results show that a training session embedded into the game, as well as the technology-savviness of the children improve the response times and the success rates of the children in an imitation game.
\end{abstract}

Keywords: Human-computer interaction, interface design, drum playing, game, multimodality, learning

\section{Introduction}

Games constitute a core element in the mental and psychological growth of children. Children improve their mental and social abilities, which are significant for them to be part of their social environments [1] [2]. Game playing has a key role for young individuals to learn their social environments and interact with other people [3]. Vygotsky emphasizes that games are a significant exercise to increase creativity and improvement of children [4]. A guideline named as "International Classification of Disabilities and Functions", which belongs to World Health Organization justifies games has an essential role in children's life [5].

Furthermore, mobile games are very popular among children. They are useful tools to introduce imitation, turn-taking, communication and memory skills as well as assisting in the school activities, as therapy and education tools. Unlike the desktop applications, mobile device applications benefit from mobility and interaction since they enable multimodality utilizing senses such as touch, sound and vision.

In this work, we present a drumming-based multimodal mobile game interface. This interface combines haptic, visual and auditory sensing to produce drumming through a mobile device. It is suggested to use in disabled young individuals' mental and psychological education within a system based on sign language education with humanoid robots. To our knowledge, there is no other application running on mobile devices with the aim of teaching children how to drum, supports multimodality and easily reachable environment as well as provides related scientific data (e.g., drumming performance, duration, and turn-taking information) gathered during gameplay for researchers.

\footnotetext{
${ }^{1}$ Istanbul Technical University, Department of Computer Engineering, Istanbul, Turkey; ORCID: 0000-0002-1144-9087

${ }^{2}$ Istanbul Technical University, Department of Computer Engineering, Istanbul, Turkey; ORCID: 0000-0003-4796-4766

${ }^{3}$ Istanbul Technical University, Department of Computer Engineering, Istanbul, Turkey; ORCID: 0000-0003-1417-8152

${ }^{4}$ Istanbul Technical University, Department of Computer Engineering, Istanbul, Turkey; ORCID: 0000-0002-0034-030X

* Corresponding Author Email: gokhan.ince@itu.edu.tr
}

\section{Related Work}

\subsection{Literature Review}

Usage of online surveys to collect information from different groups of people is a common practice [6]. Another study [7] presents a web-based survey that consists of robot videos. In this study, robots were showing some selected Turkish Sign Language words in videos. The proposed interaction was carried out by people from different educational backgrounds such as engineering students, nursery school students who had normal hearing abilities. In the first step of this survey, a human tutor showed some selected Turkish Sign Language words. Then the system requested the user to choose matching robot video from multiple selection videos that consisted of robot's expression signs. The goal of this study was to justify the achievement of a robot showing the sign language words adaptively to any human signer. Besides, different age groups were tested using a videobased study. As a novelty of this paper, we propose to use a computer game to collect data from the participants.

Computer games can be used as an intermediate layer to support the cooperation skills of the children. A desktop application called as Little Mozart intents to help children playing music besides producing data for the researchers [8]. The application provides many musical instruments to play together and was developed as part of LIREC EU FP7 project. There are more games supported in mobile platforms to improve mental and social skills of children. "Lingling Learn Spanish" aims to teach children Spanish words with fun games [9] and "my Baby Drum" presents infants first musical sounds and improves their touching skills [10].

There are some solutions provided with games to help the mental and psychological education of children having special needs. In addition to these, some studies also exist exclusively for hearing impaired children [11], such as an interactive game called CopyCat to support the education of American Sign Language [12]. The Interactive Computer Identification and Correction of Language Errors (ICICLE) project uses computer-aided commands to create a system and provides hearing impaired children special lectures and guidelines [13]. Many other applications also exist for the 
education of individuals with autism using speech therapy, imitation, turn taking and similar methods [14].

There are also projects that are based on games to improve social development of children [15]. Other studies [7], [16], in which the mobile interface was used as a communication media between the therapist/tutor, the usage of a mobile robot $\mathrm{Nao} \mathrm{H} 25$ was presented. The primitive actions of the robot were initiated using the mobile device and the interactive game between the children and the robot was able to be tailored due to each individual child's needs. The game was specially designed and tested with children having autism spectrum disorder.

\subsection{Research Questions}

This study explores the impact of mobile platforms in teaching drum playing to children coming from different social-cultural backgrounds. The project provides a proof of concept system to other researchers, which will demonstrate the capability and importance of collecting data from children to allow the researchers to analyze children's learning performance on the spot without installing expensive test setups and carefully designed experimentation environments, but by only using a mobile application in the framework of a turn-taking game. Thus, the main research questions addressed in this project are as follows:

- Is it possible to use mobile devices to gather data from children?

- Can we create a mobile device framework to test different experimental parameters with children?

- Is a mobile platform effective in the learning process of children?

- Do different social-cultural background, and technologysavviness of the children play a significant role in learning from mobile platforms/computational applications?

We expected to see that the mobile devices will be effective in the learning process of children. It was also expected that mobile devices provide a stable and fruitful test environment to the researchers.

\section{Robostar Game Project}

The main aim of the project "Robostar game" is to deploy an assistive system to be used with/by the tutor or family to teach playing the drum to children and also to gather data from them. The project focuses on teaching playing drums to children by using an amusing interface, as well as collecting the choices of the participants, namely the children in the background, the duration of the actions of children to understand their learning ability, and speed of interaction; thus it's advantages are much folds.

This is the first project of our research team on the usage of a novel interface on mobile platforms within interaction games guiding the participants/learners. By using this tool, it will be a lot easier to gather data from children. Another strength of this mobile platform is that it can be tested anywhere and send the results to the experimenters to be evaluated instantaneously.

\subsection{Design Guidelines}

Because the target group of the project is mainly researchers, who need to work especially with children to test their interactive experimental systems and perform evaluations, five interviews with skilled professionals were made to get requirements of the project among three of whom were researchers in computer science. They were expected to use the application with different profession levels. However, getting requirements just from target group researchers was not sufficient because the game will be played by children. Thus, ideas from different perspectives were necessary to distinguish features of the application, which required understanding about key aspects such as the method of thought of children, attractive activities for them, expected rewards, etc., which were collected from two more interviewees, a pre-school teacher and a designer. The highlights and important points considered in the interviews were such as below:

- Tests for children should be like a game, not an exam, thus encouraging their participation.

- Children must complete all steps and not get bored while the data is getting processed.

- User interfaces must be simple; children should not need any help while they are using the application.

- If they cannot understand what they should do next, it will discourage them and cause them to stop playing. To reinforce that, colorful pictures can be used. For instance, to ask their gender, Mickey and Minnie Mouse can be used instead of letters, cones with different amounts of ice-cream can be used to ask how much they like anything instead of asking for their scores using numbers, e.g., one to five.

- The main problem of existing test environments is their user interface: good engineering to get data is utilized, but due to poor understanding of children psychology the user experience suffers.

- The application should be developed for children aged 5-12 (kindergarten and elementary schools' students) to aim for the most rapid development of the skills to be regulated by technological solutions. Also, children should have communication abilities to some extend to receive regular and meaningful data.

- Teaching written music for drum could be difficult. Instead, some signs with particular colors could be used to show children sequence of drum beats.

- The test must be applied individually to every child to prevent impressions or cheating of other children.

- Headsets can be used during the test and it could provide making tests with more than one child at the same time, but the testing environment for each child must be the same.

- A character could be created to guide children and entertain them.

- The drum could vibrate; its color could blink; several arrows could be used to show to children, which one is a true choice.

Children like vivid designs with bright colors. Children should select their own character and the characters could have their peculiar features.

\subsection{Proposed System}

Interviews provided ideas about primary sketches and needs of projects. Researches gave feedback and with the help of interviews new version of the system is formed. Finally, a system is designed which is enjoyable for players. Besides, it collects significant data to scientists in the background. The system works by using the following steps.

\subsection{Getting user information}

The application serves just like a typical survey and collects 
demographic data from the player such as his/her name, age, gender etc. as it is shown in Figure 1. To provide a more usable and easier platform for the children, images/illustrations are chosen instead of typed texts in most suitable areas such as gender selection using buttons based on boy and girl images.

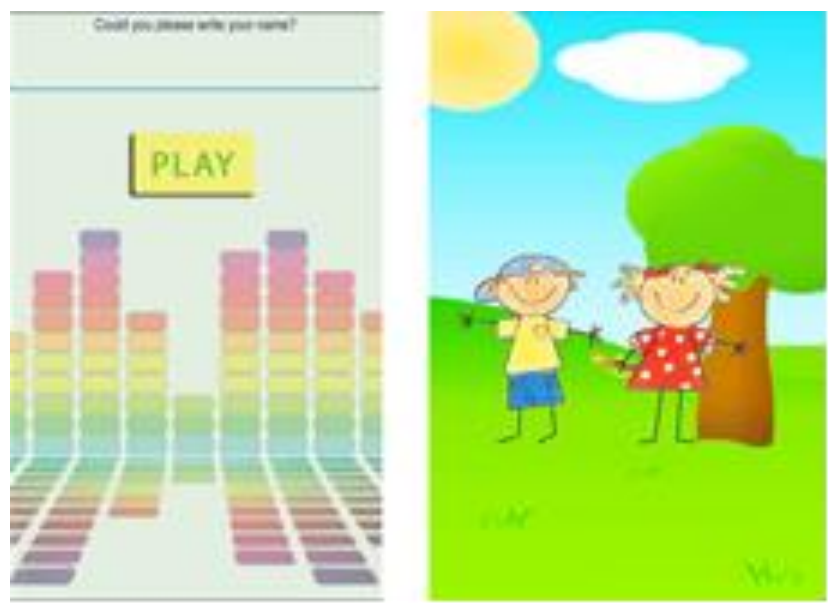

Figure 1. Screenshots from the information retrieval phase. Left side: asking name, right side: asking gender

\subsection{Selecting a Character}

After getting personal information about the user, the participant is encouraged to select a robot character to play with Figure 2. This character persists to appear on the game screen as an avatar of a robot (Figure 2 right side, top left corner) to show the order of the beats to the user during the game, and it is also depicted in the evaluation screens. Characters blink on this screen until the user selects one of them, who assists him/her throughout the game.
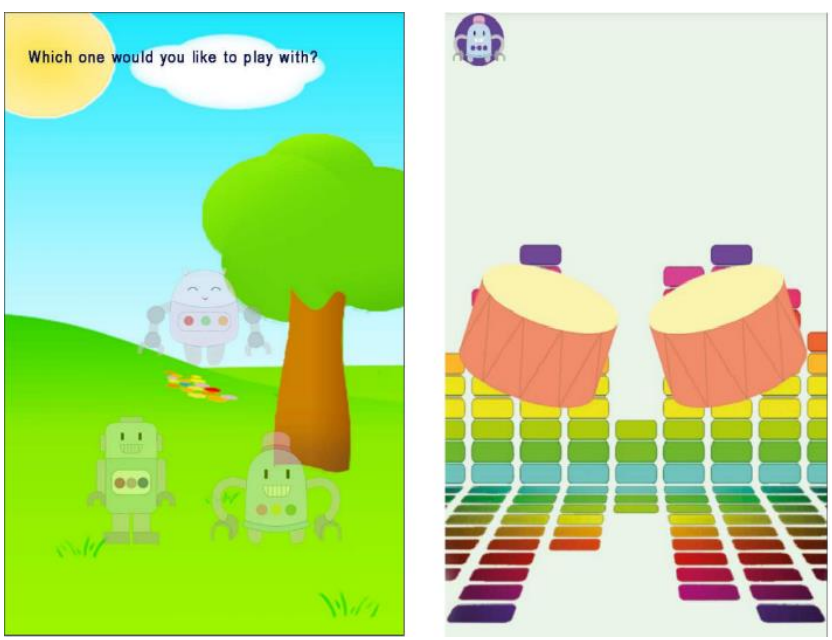

Figure 2. Screenshots from the character retrieval phase. Left side: selecting a character, right side: first level

\subsection{Playing Drums}

One of the main functions of this application is teaching kids to play drums in an interactive game. The game is adaptive to the skills of the child, i.e., the more level the child can successfully finish, the number of drums and corresponding beats start to appear as in Figure 3.

As can be seen Figure 4 each drum has its own color to stimulate the memory of the user visually as well. The drum changes its color when it is the turn of the drum respectively, or if the user selects it during his/her own turn.

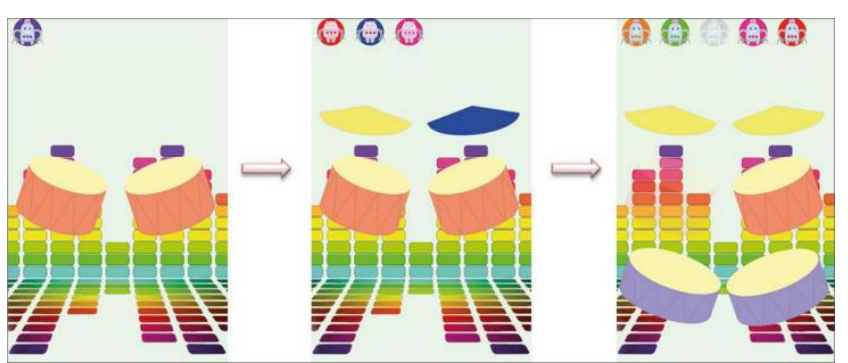

Figure 3. Different levels of the application

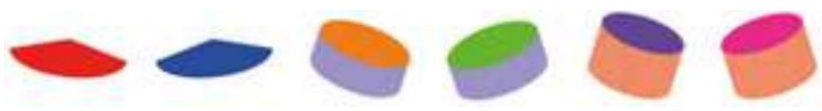

Figure 4. Active (colored) versions of the drums

To help a user, the robot character sequence shows the beat order on top of the screen. Each level has a single task, which is determined by a predefined sequence of beats. If the user plays an incorrect drum sequence, the application repeats the level until the sequence is correctly played by the user. Between each level, a feedback is displayed on the screen to indicate the success in accomplishing the given task. After finishing drum playing part, the final score of the user is shown with an ice-cream in the next screen. The amount of the ice-cream layers represents the score of the user. The height of the scores is indicated by different colors of layered ice-creams.

\subsection{Logging}

The log file is created on the local storage of the mobile device including user information and game data for each level, which is compared with the ground truth beat sequences once the game is over and is mailed to the evaluators.

\section{Experiments}

The development was performed using a tablet computer of Samsung Note 10.1, which runs on Android operating system. However, the application can run on any compatible device, which supports Android OS 3.1 or higher. Eclipse and Android Development Tool with virtual Android machines were used in the developing process because Android Development Tool includes Java and XML support to create applications. The design of the interfaces and the characters were made using Photoshop and Rhino.

In our study, we collected data from two groups of children stemming from different sociocultural backgrounds. The first group was selected from a kindergarten, which is located in a village in the underdeveloped eastern part of Turkey. These children did not have any experience on Android based mobile devices. The second group was from another kindergarten, which is located in a major metropolitan city, the capital of Turkey, Ankara. The second group had already experience on such technological devices (Table 1). Both children groups were the same age of five.

Table 1. Number of children participating in the experiments

\begin{tabular}{lcc}
\hline & $\begin{array}{c}\text { Familiar with } \\
\text { mobile devices }\end{array}$ & $\begin{array}{c}\text { Not familiar with } \\
\text { mobile devices }\end{array}$ \\
\hline Female & 3 & 6 \\
Male & 4 & 6 \\
\hline
\end{tabular}

The experiments were conducted within the supervision of their 
kindergarten teacher using a big screen smartphone and tablet computers. After running the application, writing the names of the children using the virtual keyboard and handing the mobile device over to the children, the teacher neither intervenes nor interrupts the children.

In the training session, each single drum beat of one of the six different drums is to be repeated by the participants. The training is followed by asking the children to imitate the double beats of the sequence of the drums in any given order five times, and then triple beats of the drums (again in any given order) two times. If they fail in either one of the $6+5+2=13$ sequences, the child is forced to repeat the corresponding sequence until the beats are correctly rehit. The whole training session consists of $1 \times 6+5 \times 2+2 \times 3=22$ beats in total, however the number of beats played by the child increases by each incorrect hit. In the test session, though, the children are asked to repeat a predefined pattern of four consecutive beats only once. This number, four, is heuristically selected based on the cognitive and mental capabilities of kindergarten children considering the difficulty of the given task.

This study reports the results of the presented multimodal mobile interaction game with the aim of evaluating the effect of the familiarity of technology-savviness of children. We also want to prove the effectiveness of the training session included in the game. Thus, the performance of the children in terms of the interaction duration and the success rates were assessed not only in the test session, but also in the training session. Because the children are not literate yet, we did not apply a prior and follow-up questionnaire to them.

\section{Results}

\subsection{Results of training session}

The figures presented in Table 2 are the average number of total beats performed by the children, the number of wrong beats and the response times in average. In the training session, we did not find a significant difference in the performance of the number of beats hit by the students belonging to either one of the groups, however the response time of the children having prior experience with the mobile gadgets is shorter compared to the interaction times of children having no experience at all.

Table 2. Training performance of the children in relation to their familiarity with the mobile devices

\begin{tabular}{lcc}
\hline & $\begin{array}{c}\text { Familiar with } \\
\text { mobile devices }\end{array}$ & $\begin{array}{c}\text { Not familiar with } \\
\text { mobile devices }\end{array}$ \\
\hline $\begin{array}{l}\text { Number of total } \\
\text { beats }\end{array}$ & $43.25 \pm 17.7$ & $45.71 \pm 16.3$ \\
$\begin{array}{l}\text { Number of } \\
\text { incorrect beats }\end{array}$ & $16.83 \pm 13.97$ & $19.71 \pm 13.45$ \\
Response time [sec] & $2.86 \pm 0.54$ & $3.30 \pm 1.47$ \\
\hline
\end{tabular}

\subsection{Results of test session}

In the test phase, however, the familiarity plays a drastic role in improving the correct rates (by around 0.82 beats) as well as reducing the mean duration of response of the children (by around 0.65 seconds) as shown in Table 3 . This result indicates that the training session helped both groups of children to improve their response times by approximately 1.1 to 1.3 seconds. The standard deviation of the more technology-savvy children's response became even shorter demonstrating the fact that the design of the training phase of the application was useful for the children's faster comprehension of the problem and easier adaptation to the given task.
Table 3. Test performance of the children in relation to their familiarity with the mobile devices

\begin{tabular}{lcc}
\hline & $\begin{array}{c}\text { Familiar with } \\
\text { mobile devices }\end{array}$ & $\begin{array}{c}\text { Not familiar with } \\
\text { mobile devices }\end{array}$ \\
\hline $\begin{array}{l}\text { Number of correct } \\
\text { beats }\end{array}$ & $2.25 \pm 1.42$ & $1.43 \pm 1.40$ \\
Response time [sec] & $1.56 \pm 0.27$ & $2.21 \pm 1.27$ \\
\hline
\end{tabular}

\subsection{Discussion}

In the follow-up conversation with children, who had the chance to play the game later at home in their own mobile devices, we learned that they have grown a habit of playing the game and achieving perfect scores just by following the robot pictures on the screen and ignoring the sounds as well as the drum animations. This may suggest that using an increased number of modalities and stimuli in a game on a multimodal device like the tablets and smartphones can cause confusion on the small children rather than providing additional cues, which we contrarily believed would have contributed to their success rates on the task.

\section{Summary and Outlook}

In this study, we presented a design framework to build a multimodal mobile game for children to play drums interactively and for researchers to gather data about their test subjects. We presented the results of this interaction game with the aim of evaluating the effect of the familiarity of technology-savviness of the children using the outcomes of a learning/memory game played on a mobile device. We also showed the effectiveness of the training session to enhance the performance of the children in terms of the interaction duration and the success rates. The performance of the children in test session was improved by approximately 1.1 to 1.3 seconds in their response time.

Some future work on this project will be to attempt to improve the usability of the core functionalities through extensive user testing on our target audience. Our previous tests did not include hearing impaired children in our tests so we could not evaluate the usability of the system on our core user group. We will also enhance the accessibility of the application for the hearing impaired. Currently the system supports audio and visual modes of output to produce feedback. However, hearing impaired users receive only the visual feedback. To complement the audio part, we plan to add haptic feedback in the form of vibration. For this task we are going to research the usage of haptic feedback in mobile applications and map different vibrations to correspond with different audio outputs such as different notes and "success" or "failure" system messages in game modules.

\section{References}

[1] Spitzer, S. L., "Play in children with autism: Structure and experience" in Play in Occupational Therapy for Children, (2nd ed.), Mosby Elsevier, St. Louis, MO, pp. 351-374, 2008.

[2] Iacono, I., Lehmann, H., Marti, P., Robins, B., Dautenhahn, K., "Robots as social mediators for children with autism - A preliminary analysis comparing two different robotic platforms", IEEE International Conference on Development and Learning (ICDL), pp. 1-6, 2011.

[3] Besio, S., "Analysis of Critical Factors Involved in Using in Interactive Robots for Education and Therapy of Children with Disabilities", UNIService, Trento, 2008.

[4] Vygotsky, L, "Mind in Society", Harvard University Press, Cambridge MA., 1978.

[5] WHO, "International Classification of Functioning, Disability and 
Health”, Geneva: World Health Organization, 2001.

[6] Andrews, D., Nonnecke, B. and Preece, J., "Conducting research on the internet: Online survey design, development and implementation guidelines", International Journal of Human Computer Interaction, 16(2), pp. 185-210, 2003.

[7] Kose, H., Yorganci, R., Algan H. E., Syrdal D.S. "Evaluation of the robot assisted sign language tutoring using video-based studies", International Journal of Social Robotics, Volume 4, Number 3, 273283, 2012.

[8] Little Mozart 2 - LIREC Project, [Online]. Available: http://mozart.imagina.pt, Accessed on: Nov. 7, 2019.

[9] LingLing Language Learning Game, [Online]. Available: http://www.joinlingling.com/, Accessed on: Nov. 7, 2019.

[10] MyBabyDrum Android Application, [Online]. Available: http://www.dooet.com/android/My-baby-drum-3376814129 Accessed on: Nov. 7, 2019.

[11] Adamo-Villani, N., "A virtual learning environment for deaf children: Design and evaluation", International Journal of Applied Science, Engineering, and Technology 16, 18-23, 2006.

[12] Weaver, K. A., Hamilton, H., Zafrulla, Z., Brashear, H., Starner, T., Presti, P., Bruckman, A. "Improving the language ability of deaf signing children through an interactive American Sign Languagebased video game", 9th International Conference of the Learning Sciences, 306-307, 2010.

[13] Greenbacker, C., McCoy, K., "The ICICLE Project: An Overiew”, First Annual Computer Science Research Day, Department of Computer and Information Sciences, University of Delaware, 2008.

[14] Autismspeaks Mobile Application, [Online]. Available: http://www.autismspeaks.org/autism-apps, Accessed on: Nov. 7, 2019.

[15] Hakkarainen, P., "Play and motivation", in Perspectives on Activity Theory, Y. Engestrm, R. Meittinen and R. Punamaki (eds), Cambridge University Press, Cambridge, UK, 2003.

[16] Ertugrul, B.S., Kivrak, H., Daglarli, E., Kulaglic, A., Tekelioglu, A., Kavak, S., Ozkul, A., Yorganci, R., Kose,H. "iSign: Interaction games for humanoid assisted sign language tutoring", International workshop on human-agent interaction, 2012. 